\title{
•生物编目・
}

\section{蕨类植物PPG I系统与中国石松类和蕨类植物分类}

\author{
张丽兵 ${ }^{*}$ \\ (Missouri Botanical Garden, P. O. Box 299, St. Louis, Missouri 63166-0299, USA)
}

\section{The PPG I classification and pteridophytes of China}

Libing Zhang ${ }^{*}$

Missouri Botanical Garden, P. O. Box 299, St. Louis, Missouri 63166-0299, USA

广义蕨类植物 PPG I (Pteridophyte Phylogeny Group I) 分类系统的出版(PPG I, 2016), 使得全世界 偋类植物学工作者自 Smith 等(2006)分类系统 10 年 之后, 首次有了全面的最新指南, 其重大意义不言 而喻。

与 Smith 等(2006)、Christenhusz 等(2011)及 Christenhusz 和 Chase (2014) 的分类系统不同, PPG I 真正算是集体智慧的结晶。PPG I 由 Eric Schuettpelz 博士和 Harald Schneider 博士策划。在形成共识之 前, 来自全世界 25 个单位的 26 位组织者分别组织 了一个(或多个)有争议的较大的类群(一般为科)的 小组讨论, 先由这些组织者在感兴趣的小组内对某 一类群达成共识后, 再提交给 PPG I 的总组织者 Eric Schuettpelz 博士, 由 Eric 发送给大家讨论, 最 后根据大家的意见修改后通过。PPG I 论文由来自 全世界 68 个单位的 94 名作者联合署名发表, 包括 20 名华人藓类学家。

PPG I 沿袭了以前许多蒝类分类系统的大概念 的传统(如 Kramer \& Green, 1990; Smith et al, 2006; Christenhusz et al, 2011; Christenhusz \& Chase, 2014), 将全世界现存约 12,000 种石松类和偋类植物分成 2 纲 14 目 51 科 337 属。石松类和蕨类植物的种类与 藓类植物的种类相当, 但藓类学家承认 112 个或更 多个科、845 或更多个属(Crosby et al, 1999; Buck \& Goffinet, 2000; Goffinet \& Buck, 2004)。大科和大属 的概念有利于这些类群的稳定性, 因为它们的单系
性不容易受单个新研究的影响。但大科和大属的概 念也有不利的一面, 例如可能会使得硕类学家申请 经费(例如跟藓类学家竞争时)受影响, 因为他们研 究的类群可能只是一个属下的组而不是一个属。另 外, 对保育研究者和工作者来说, 大科和大属的概 念可能使研究的类群因此而变成一个低等级的类 群而不受政策制定者、管理者或项目审阅者的重 视。实际上, 石松类和蕨类植物中大科和大属的概 念反映了石松类和蓱类植物形态上的复杂性, 并暗 示茨类学家对许多类群内和类群间的系统关系还 知之甚少, 还需深入研究。

\section{PPG I与FOC薜类分类的比较}

Flora of China (FOC) (2-3 卷, 蕨类; Lin et al, 2013) 是全世界第一个在科一级水平上采用基于现 代分子系统学结果的分类系统的硕类植物志。PPG I 分类系统与 $\mathrm{FOC}$ 在科一级水平上的处理基本一致, 但 PPG I 对一些属的处理与 FOC 不同, 具体如下:

石松科(Lycopodiaceae): 将该科分成 3 个亚科, 即石松亚科 (Lycopodioideae) 、小石松亚科 (Lycopodielloideae)和石杉亚科(Huperzioideae)。在 属一级, PPG I 恢复了 3 个 《中国植物志》第 6(3)卷 采用(张丽兵, 2004)但 FOC (Zhang \& Iwatsuki, 2013) 不承认的属, 即扁枝石松属(Diphasiastrum)、拟小石 松属(Pseudolycopodiella)和垂穗石松属(Palhinhaea); 增加了单穗石松属 (Spinulum) 和笔直石松属 
(Dendrolycopodium) (也见 Zhou \& Zhang, 2017)。 紫萁科(Osmundaceae): PPG I 建立了 1 个新属, 即线紫萁属(Claytosmunda)。

凤尾硕科(Pteridaceae): PPG I 中, 一条线硕属 (Monogramma)被合并到书带䓲属(Haplopteris)中。

冷硕科 (Cystopteridaceae): 光 叶䓲属 (Cystoathyrium)被合并到冷蒴属(Cystopteris)中。

金星蕨科(Thelypteridaceae): 将该科分为 2 个 亚科, 金星硕亚科(Thelypteridoideae)和卵果蒴亚科 (Phegopteridoideae)。茯蒝属(Leptogramma)和圣莜属 (Dictyocline) 被合并到溪边蕨属(Stegnogramma)中。

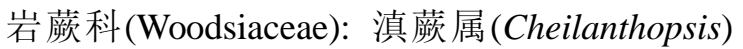
和膀胱蒝属 (Protowoodsia) 被合并到岩硕属 (Woodsia)中。

乌毛蕨科 (Blechnaceae): 崇 澍硕属 (Chieniopteris) 被合并到狗脊属(Woodwardia)中。

蹄盖蕨科(Athyriaceae): 安䕬属(Anisocampium) 和角硕属(Cornopteris)被合并到蹄盖硕属(Athyrium)。

鳞毛硕科(Dryopteridaceae): 新增加了 1 个亚科, 即毛脉䕆亚科(Polybotryoideae) (Liu et al, 2016), 新 增加了 1 属, 即毛脉烣属(Trichoneuron)。黄腺羽蒝 属(Pleocnemia)被从三叉䓲科(Tectariaceae)中移到鳞 毛硕科中。

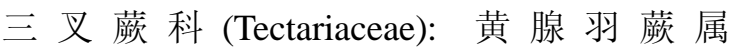
(Pleocnemia) 被移到鳞毛蒝科中。

骨碎补科 (Davalliaceae): 假钻毛蕨属 (Paradavallodes)、小膜盖蕨属(Araiostegia)和阴石蕨 属(Humata)被合并到骨碎补属(Davallia)中。全科现 在只含 1 个属。

水龙骨科(Polypodiaceae)：该科变化很大，被分 为 6 个亚科, 即剑䓲亚科(Loxogrammoideae)、水龙骨 亚科(Polypodioideae)、鹿角葓亚科(Platycerioideae)、 檞蓱亚科(Drynarioideae)、星烣亚科(Microsoroideae)

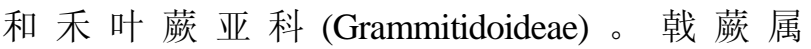
(Christiopteris) 、槲葓属 (Drynaria) 和顶育硕属 (Photinopteris)被合并到连珠蔽属(Aglaomorpha)中。篦 齿蒝属(Metapolypodium)、拟水龙骨属(Polypodiastrum) 和水龙骨属 (Polypodiodes) 被合并到棱脉蒝属 (Goniophlebium)中。锡金假瘤藃属(Himalayopteris)被 合并到修蒝属(Selliguea)中。高平硕属(Caobangia)被 合并到伏石硕属 (Lemmaphyllum) 中。瘤蒝属 (Phymatosorus)被合并到星颌属(Microsorum)中。
另外, PPG I 增加了由笔者和张良博士最近发 表的翼囊蒝科(Didymochlaenaceae) (Zhang \& Zhang, 2015)。其模式种近年也在我国西双版纳发现有分布 (Tan et al, 2015)。该科由秦仁昌先生(Ching, 1940) 最先提出但未正式发表。

\section{2 中国学者在蕨类植物科、属一级的贡献}

在科级水平上, PPG I 共接受 51 科, 其中中国 学者发表的有翼囊蕨科(Zhang \& Zhang, 2015)、肿 足蒝科(Hypodematiaceae; Ching, 1975)、轴果硕科 (Rhachidosoraceae; Christenhusz et al, 2011)和肠蒴 科(Diplaziopsidaceae; Christenhusz et al, 2011)。在属 级水平上, PPG I 共接受 337 个属, 其中中国学者参 与发表的属有 21 个, 包括秦仁昌先生发表的 17 个 (包括 5 个新等级)、邢公侠先生发表的金毛裸藃属 (Paragymnopteris) (Shing, 1993)、秦仁昌与邢公侠先 生合作发表的鳞果星偋属(Lepidomicrosorium) (秦 仁昌和邢公侠, 1983)、笔者和张良博士发表的龙蕨 属(Draconopteris) (Zhang et al, 2016)，以及笔者与 Schuettpelz 发表的马来蕨属(Malaifilix) (Zhang et al, 2016)。

秦仁昌先生发表的被 PPG I 承认的 17 个属(包 含 5 个新等级)包括:

美洲里白属(Gleichenella Ching)（以下原始文 献引证略; Gleicheniaceae);

轴果䕬属(Rhachidosorus Ching) (Rhachidosoraceae); 针毛蕨属 (Macrothelypteris (H. Ito) Ching) (Thelypteridaceae);

紫柄蒝属(Pseudophegopteris Ching) (Thelypteridaceae);

方 杆 蕨 属 (Glaphyropteridopsis Ching) (Thelypteridaceae);

龙津蒴属(Mesopteris Ching) (Thelypteridaceae);

凸轴蕨属 (Metathelypteris (H. Ito) Ching) (Thelypteridaceae);

金星蕨属 (Parathelypteris (H. Ito) Ching) (Thelypteridaceae);

假毛蕨属(Pseudocyclosorus Ching) (Thelypteridaceae);

毛脉蕨属(Trichoneuron Ching) (Dryopteridaceae); 节毛硕属(Lastreopsis Ching) (Dryopteridaceae); 拟耳䒴欠属(Parapolystichum (Keyserl.) Ching) 
(Dryopteridaceae，中国不产);

牙蕨属(Pteridrys C. Chr. \& Ching) (Tectariaceae); 鳞果星䕋属(Lepidomicrosorium Ching \& $\mathrm{K} . \mathrm{H}$.

Shing) (Polypodiaceae);

瓦韦属(Lepisorus (J. Sm.) Ching) (Polypodiaceae); 盾䕬属(Neolepisorus Ching) (Polypodiaceae); 毛鳞莜属(Tricholepidium Ching) (Polypodiaceae)。

除了发表的新类群被接受外, 众多华人学者近 年来发现的新的系统关系也被 PPG I 系统接受, 特 别是大量以中国为分布中心的类群的系统关系得 到了 PPG I 系统的承认。但总体来说, 虽然中国蓱 类植物种类众多, 近年来也开展了大量的系统发育 研究工作, 但是华人学者在 PPG I 系统的发表过程 中的主导作用有限。

致谢: 本文写作中得到梁燕女士的多方帮助。周新 茂博士帮助输入科、属中文名。感谢审稿人的建议。

\section{参考文献}

Buck WR, Goffinet B (2000) Morphology and classification of the mosses. In: Bryophyte Biology (eds Shaw AJ, Goffinet B), pp. 71-123. Cambridge University Press, Cambridge.

Ching RC (1940) On natural classification of the family "Polypodiaceae". Sunyatsenia, 5, 1-37.

Ching RC (1975) Two new fern families. Acta Phytotaxonomica Sinica, 13, 96-98. (in Chinese with English abstract) [秦仁昌 (1975) 萍类植物的两新科. 植物分 类学报, 13, 96-98.]

Ching RC, Shing KH (1983) Lepidomicrosorium Ching et Shing, a new fern genus of Polypodiaceae from China. Botanical Research: contributions from the Institute of Botany, Academica Sinica, 1, 1-14. (in Chinese with English abstract) [秦仁昌, 邢公侠 (1983) 鳞果星蕨属一一中国水 龙骨科的一个新属. 见: 植物学集刊(第1集), 1-14页.]

Christenhusz MJM, Chase MW (2014) Trends and concepts in fern classification. Annals of Botany, 113, 571-594.

Christenhusz MJM, Zhang X-C, Schneider H (2011) A linear sequence of extant families and genera of lycophytes and ferns. Phytotaxa, 19, 7-54.

Crosby MR, Magill RE, Allen B, He S (1999) Checklist of Mosses. Missouri Botanical Garden, St. Louis.

Goffinet B, Buck WR (2004) Systematics of the bryophyta (mosses): from molecules to a revised classification. In: Molecular Systematics of Bryophytes (eds Goffinet B, Hollowell V, Magill R), pp. 205-239. Missouri Botanical Garden Press, St. Louis.

Kramer KU, Green PS (1990) Pteridophytes and gymnosperms.
In: The Families and Genera of Vascular Plants, Vol. 1 (ed. Kubitzki K), pp. 1-404. Springer, Berlin, Heidelberg \& New York.

Lin Y-X, Zhang L-B, Zhang X-C, He Z-R, Wang Z-R, Lu S-G, Wu S-G, Xing F-W, Zhang G-M, Liao W-B, Xiang J-Y, Wang F-G, Qi X-P, Yan Y-H, Ding M-Y, Liu J-X, Dong S-Y, He H, Zhang Q-Y, Moore S-J, Wu Z-H, Li Z-Y, Jin X-F, Ding B-Y, Liu Q-R, Shi L, Barrington DS, Kato M, Iwatsuki K, Gilbert MG, Hovenkamp PH, Nooteboom HP, Prado J, Viane R, Christenhusz MJM, Yatskievych G, Ebihara A, Serizawa S, Parris BS, Ranker TA, Sahashi N, Hooper EA, Barcelona J, Shmakov A, Nishida H, Lin S-J, Smith AR, Funston AM, Haufler C, Turland NJ, Hanks JG, Mickel JT, Kadokawa Y, Pryer KM, Taylor WC, Johnson DM, Alverson ER, Metzgar JS, Masuyama S (2013) Pteridophytes. In: Flora of China, Vol. 2-3 (eds Wu ZY, Raven PH, Hong DY), pp. 1-959. Science Press, Beijing \& Missouri Botanical Garden Press, St. Louis.

Liu H-M, Zhang X-C, Wang M-P, Shang H, Zhou S-L, Yan Y-H, Wei X-P, Xu W-B, Schneider H (2016) Phylogenetic placement of the enigmatic fern genus Trichoneuron informs on the infra-familial relationship of Dryopteridaceae. Plant Systematics and Evolution, 302, 319-332.

PPG I (2016) A community-derived classification for extant lycophytes and ferns. Journal of Systematics and Evolution, 54, 563-603.

Shing K-H (1993) A new genus, Paragymnopteris Shing separated from Gymnopteris Bernh. Indian Fern Journal, 10, 226-231.

Smith AR, Pryer KM, Schuettpelz E, Korall P, Schneider H, Wolf PG (2006) A classification for extant ferns. Taxon, 55, 705-731.

Tan Y-H, Wei R, Li J-W, Zhang X-C (2015) Didymochlaena Desv. (Hypodematiaceae): a newly recorded fern genus to China. Plant Diversity \& Resources, 37, 135-138.

Zhang L, Schuettpelz E, Rothfels C, Zhou X-M, Gao X-F, Zhang L-B (2016) Circumscription and phylogeny of the fern family Tectariaceae based on plastid and nuclear markers, with the description of two new genera: Draconopteris and Malaifilix (Tectariaceae). Taxon, 65, 723-738.

Zhang L-B (2004) Lycopodiaceae. In: Flora Reipublicae Popularis Sinicae, Tomus 6(3) (ed. Wu ZY), pp. 55-85. Science Press, Beijing. (in Chinese) [张丽兵 (2004) 石松 科. 见: 中国植物志第6卷(3) (吴征镒主编), 55-85页. 科 学出版社, 北京.]

Zhang L-B, Iwatsuki K (2013) Lycopodiaceae. In: Flora of China, Vol. 2-3 (eds Wu ZY, Raven PH, Hong DY), pp. 13-36. Science Press, Beijing \& Missouri Botanical Garden Press, St. Louis.

Zhang L-B, Zhang L (2015) Didymochlaenaceae-A new fern family of eupolypods I (Polypodiales). Taxon, 64, 27-38.

Zhou X-M, Zhang L-B (2017) Dendrolycopodium verticale comb. nov. (Lycopodiopsida: Lycopodiaceae) from China. Phytotaxa, 295, 199-200.

(责任编委: 严岳鸿 责任编辑: 时意专)

\section{附录 Supplementary Material}

附录1 中国石松类和蕨类植物科、亚科、属列表

Appendix 1 List of families, subfamilies, and genera of lycophytes and ferns of China

http://www.biodiversity-science.net/fileup/PDF/2016372-1.pdf 
附录 1 中国石松类和蔽类植物科、亚科、属列表 (科名按 FOC 的顺序排列, 括号内为 FOC 页码, 中文斜 体的属为异名, 下划线表明为 PPG I 新增加而 FOC 中没有的类群, 中名依据原类群等级的中名或惯用法拟 定, 建议规范使用)。

Appendix 1 List of families, subfamilies, and genera of lycophytes and ferns of China (The order of families follows that in FOC. Page numbers in parentheses denote those page numbers in FOC. italic Chinese generic names indicate synonyms. Underlined names indicate added names from the PPG I which were not in FOC. Chinese names follow original names adopted in FOC or are coined following tradition).

Lycopodiaceae 石松科 (p. 13)

Huperzioideae 石杉亚科

1. Huperzia 石杉属

2. Phlegmariurus 马尾杉属

Lycopodielloideae 小石松亚科

3. Lycopodiella 小石松属

4. Palhinhaea 灯笼石松属

Lycopodioideae 石松亚科

5. Dendrolycopodium 笔直石松属

6. Diphasiastrum 扁枝石松属

7. Lycopodium 石松属

8. Lycopodiastrum 藤石松属

9. Pseudolycopodiella 拟小石松属

10. Spinulum 单穗石松属

Isoëtaceae 水非科 (p. 35)

1. Isoëtes 水非属

Selaginellaceae 卷柏科 (p. 37)

1. Selaginella 卷柏属

Equisetaceae 木贼科 (p. 67)

1. Equisetum 木贼属

Ophioglossaceae 瓶尔小草科 (p. 73)

1. Botrychium 阴地烣属

2. Helminthostachys 七指偋属

3. Ophioglossum 瓶尔小草属

Psilotaceae 松叶蕨科 (p. 81)

1. Psilotum 松叶硕属

Marattiaceae 合囊萨科 (p. 82)

1. Angiopteris 莲座蕨属

2. Christensenia 天星偋属

\section{Ptisana 合囊蕨属}

Osmundaceae 紫其科 (p.90)

1. Claytosmunda 线紫萁属

2. Osmunda 紫萁属

3. Osmundastrum 桂皮紫其属

Hymenophyllaceae 膜蕨科 (p. 93)

1. Abrodictyum 长片䓲属

2. Callistopteris 毛杆蕨属

3. Cephalomanes 厚叶喏属

4. Crepidomanes 假脉蕨属

5. Didymoglossum 毛边蕨属

6. Hymenophyllum 膜葓属

7. Vandenboschia 瓶蕨属

Gleicheniaceae 里白科 (p. 110)

1. Dicranopteris 芒萁属

2. Diplopterygium 里白属

3. Sticherus 假芒萁属

Dipteridaceae 双扇蕨科 (p. 116)

1. Cheiropleuria 燕尾䓲属

2. Dipteris 双扇䓲属

Lygodiaceae 海金沙科 (p. 118)

1. Lygodium 海金沙属

Schizaeaceae 莎草蕀科 (p. 122)

1. Schizaea 莎草硕属

Marsileaceae 蘋科 (p. 123)

1. Marsilea 蘋属

Salviniaceae 槐叶苹科 (p. 125)

1. Azolla 满江红属

2. Salvinia 槐叶萍属 
张丽兵. 茨类植物PPG I系统与中国石松类和蕨类植物分类. 生物多样性, 2017, 25 (3): 340-342. http://www.biodiversity-science.net/fileup/PDF/2016315

Plagiogyriaceae 瘤足蕨科 (p. 128)

1. Plagiogyria 瘤足蕨属

Cibotiaceae 金毛狗蕨科 (p. 132)

1. Cibotium 金毛狗偋属

Cyatheaceae 杪椤科 (p. 134)

1. Alsophila 杪椤属

2. Sphaeropteris 白杪椤属

Lindsaeaceae 鳞始硕科 (p. 139)

1. Lindsaea 鳞始烣属

2. Odontosoria 乌蕨属

3. Osmolindsaea 香鳞始硕属

4. Tapeinidium 达边硕属

Dennstaedtiaceae 碗䔊科 (p. 147)

1. Dennstaedtia 碗蒝属

2. Histiopteris 栗葓属

3. Hypolepis 姬硕属

4. Microlepia 鳞盖穙属

5. Monachosorum 稀子硕属

6. Paesia 曲轴蕨属

7. Pteridium 蕨属

Pteridaceae 凤尾蕨科 (p. 169)

Cheilanthoideae 碎米蕨亚科

1. Aleuritopteris 粉背硕属

2. Calciphilopteris 戟叶黑心蒝属

3. Cheilanthes 碎米硕属

4. Doryopteris 黑心莏属

5. Parahemionitis 泽泻硕属

6. Paragymnopteris 金毛裸蒴属

7. Pellaea 旱硕属

Cryptogrammoideae 珠蕨亚科

8. Coniogramme 凤了偋属

9. Cryptogramma 珠硕属

Parkerioideae 水偋亚科 (FOC中采用 Ceratopteridoideae这一名称)

10. Acrostichum 卤葓属

11. Ceratopteris 水葓属
Pteridoideae 凤尾蕨亚科

12. Anogramma 翠蕨属

13. Pityrogramma 粉叶蕨属

14. Onychium 金粉硕属

15. Pteris 凤尾硕属

16. Taenitis 竹叶硕属

Vittarioideae 书带蕨亚科

17. Adiantum 铁线穙属

18. Antrophyum 车前硕属

19. Haplopteris 书带鄀属

Monogramma 一条线䓲属 $=$ Haplopteris

Cystopteridaceae 冷蔴科 (p. 257)

1. Acystopteris 亮毛蒝属

2. Cystopteris 冷蕨属

Cystoathyrium 光叶䓲属 = Cystopteris

3. Gymnocarpium 羽节蕨属

Aspleniaceae 铁角䓲科 (p. 267)

1. Asplenium 铁角䓲属

2. Hymenasplenium 膜叶铁角蕨属

Diplaziopsidaceae 肠鄀科 (p. 317)

1. Diplaziopsis 肠偋属

Thelypteridaceae 金星蕨科 (p. 319)

Phegopteridoideae 卵果蒝亚科

1. Macrothelypteris 针毛鄀属

2. Phegopteris 卵果蕨属

3. Pseudophegopteris 紫柄萨属

Thelypteridoideae 金星蕨亚科

4. Ampelopteris 星毛蕨属

5. Craspedosorus 边果蕨属

6. Cyclogramma 钩毛硕属

7. Cyclosorus 毛蕨属

8. Glaphyropteridopsis 方杆蕨属

9. Mesopteris 龙津蕨属

10. Metathelypteris 凸轴偋属

11. Oreopteris 假鳞毛蕨属

12. Parathelypteris 金星硕属

13. Pronephrium 新月蕨属

14. Pseudocyclosorus 假毛蕨属 
张丽兵. 蕨类植物PPG I系统与中国石松类和蕨类植物分类. 生物多样性, 2017, 25 (3): 340-342. http://www.biodiversity-science.net/fileup/PDF/2016315

15. Stegnogramma 溪边硕属

Dictyocline 圣䓲属 $=$ Stegnogramma

Leptogramma 茯䓲属 = Stegnogramma

16. Thelypteris 沼泽蕨属

\section{Didymochlaenaceae 翼囊薕科}

1. Didymochlaena 翼囊蒝属

Woodsiaceae 岩硕科 (p. 397)

1. Woodsia 岩硕属

Protowoodsia 膀胱蒝属 $=$ Woodsia

Cheilanthopsis 滇蒴属 = Woodsia

Rhachidosoraceae 轴果鄀科 (p. 405)

1. Rhachidosorus 轴果葓属

Onocleaceae 球子茨科 (p. 408)

1. Matteuccia 荚果葓属

2. Onoclea 球子蕨属

3. Pentarhizidium 东方荚果䓞属

Blechnaceae 乌毛蕨科 (p. 411)

1. Blechnidium 乌木硕属

2. Blechnum 乌毛媭属

3. Brainea 苏铁硕属

4. Diploblechnum 扫把蕨属

5. Stenochlaena 光叶藤䓲属

6. Struthiopteris 荚囊葓属

7. Woodwardia 狗脊属

Chieniopteris 崇澍䓲属 = Woodwardia

\section{Athyriaceae 蹄盖鄀科 (p. 418)}

1. Athyrium 蹄盖烣属

Cornopteris 角蕨属 = Athyrium

Anisocampium 安蔴属 = Athyrium

2. Deparia 对囊硕属

3. Diplazium 双盖蕨属

Hypodematiaceae 肿足硕科 (p. 535)

1. Hypodematium 肿足蕨属

2. Leucostegia 大膜盖蕨属

Dryopteridaceae 鳞毛蕨科 (p. 541)

Dryopteridoideae 鳞毛蕨亚科
1. Arachniodes 复叶耳硕属

2. Ctenitis 肋毛硕属

3. Cyrtomium 贯众属

4. Dryopteris 鳞毛葓属

5. Lastreopsis 节毛偋属

6. Polystichum 耳硕属

Elaphoglossoideae 舌蕨亚科

7. Bolbitis 实硕属

8. Elaphoglossum 舌偋属

9. Lomagramma 网藤烣属

10. Pleocnemia 黄腺羽烣属 (从Tectariaceae中移来)

11. Teratophyllum 符藤偋属

Polybotryoideae 毛脉屏亚科

12. Trichoneuron 毛脉䓲属

Lomariopsidaceae 藤蒴科 (p. 725)

1. Cyclopeltis 拟贯众属

2. Lomariopsis 藤蘐属

Nephrolepidaceae 肾蕨科 (p. 727)

1. Nephrolepis 肾硕属

Tectariaceae 三叉蕨科 (p. 730)

1. Arthropteris 爬树硕属

Pleocnemia 黄腺羽蕨属 (移到Dryopteridaceae)

2. Pteridrys 牙硕属

3. Tectaria 叉蕨属

Oleandraceae 条蕨科 (p. 747)

1. Oleandra 条硕属

Davalliaceae 骨碎补科 (p. 749)

1. Davallia 骨碎补属

Paradavallodes 假钻毛蒴属=Davallia

Araiostegia 小膜盖蔴属 = Davallia

Humata 阴石痰属 = Davallia

Polypodiaceae 水龙骨科 (p. 758)

Drynarioideae 葪蕨亚科

1. Aglaomorpha 连珠硕属

Photinopteris 顶育荻属 = Aglaomorpha

Drynaria 葪荻属 = Aglaomorpha 
张丽兵. 蕨类植物PPG I系统与中国石松类和蕨类植物分类. 生物多样性, 2017, 25 (3): 340-342.

http://www.biodiversity-science.net/fileup/PDF/2016315

Christopteris 戟痰属 = Aglaomorpha

2. Arthromeris 节肢蒝属

3. Gymnogrammitis 雨薜属

4. Selliguea 修蕨属

Himalayopteris 锡金假瘤荻属 $=$ Selliguea

Grammitidoideae 禾叶蒝亚科

5. Calymmodon 荷包蕨属

6. Chrysogrammitis 金禾蕨属

7. Ctenopterella 小蒿蕨属

8. Dasygrammitis 毛禾偋属

9. Micropolypodium 锯蘐属

10. Oreogrammitis 滨禾烣属

11. Prosaptia 穴子硕属

12. Radiogrammitis 辐禾硕属

13. Themelium 蒿硕属

14. Tomophyllum 裂禾蕨属

15. Scleroglossum 革舌偋属

16. Xiphopterella 剑羽偋属

Microsoroideae 星嵚亚科

17. Lemmaphyllum 伏石蕨属

Caobangia 高平痥属 = Lemmaphyllum
18. Lepidomicrosorium 鳞果星蒝属

19. Lepisorus 瓦韦属

20. Leptochilus 薄唇蕨属

21. Microsorum 星硕属

Phymatosorus 瘤蒴属 = Microsorum

22. Neocheiropteris 扇偋属

23. Neolepisorus 盾䓲属

24. Tricholepidium 毛鳞蕨属

Loxogrammoideae 剑蒝亚科

25. Loxogramme 剑䓲属

Platycerioideae 鹿角蓱亚科

26. Pyrrosia 石韦属

27. Platycerium 鹿角蒴属

Polypodioideae 水龙骨亚科

28. Goniophlebium 棱脉葓属

Metapolypodium 篦齿茨属 = Goniophlebium

Polypodiastrum 拟水龙骨属 = Goniophlebium

Polypodiodes 水龙骨属 = Goniophlebium

29. Pleurosoriopsis 睫毛硕属

30. Polypodium 多足硕属 